\title{
STUDY OF MORPHOLOGICAL AND ANGIOGENIC FEATURES OF TRIPLE NEGATIVE TUMORS BY ULTRASONOGRAPHY
}

Felipe Cordeiro Fonseca', Eduardo Carvalho Pessoa', Carla Priscila Kamyia Pessoa', Benedito Sousa Almeida Filho, Heloisa Maria De Luca Vespoli

${ }^{1}$ Faculdade de Medicina de Botucatu - Botucatu (SP), Brazil.

Introduction: Since 2013, after the Saint Gallen International Breast Cancer Conference, breast cancer is classified by its morphological, biological and prognostic features into three subgroups according to the immunohistochemichal expression, which is similar to the genetic expression of the tumor. These groups, known as luminal, HER2 enriched and triple negative, are classified according to the positivity or negativity of nuclear receptors (estrogen [ER] and progesterone receptors [PR]) or membrane receptors (HER2). Triple negative tumors do not express positivity for any of these receptors. This group is composed of tumors with a more adverse biology and more aggressive behavior, affecting women in a younger age than other groups, and they also have higher rates of recurrence and lower overall survival. Objectives: This study aims to assess morphological and angiogenic features of these tumors by ultrasound exams. Methods: This is a prospective study, in which 129 lesions with an immunochemistry study compatible with triple negative tumors were selected from 1,180 breast carcinomas diagnosed by ultrasound and core biopsy from 2012 to 2017. Features assessed were shape, margin, posterior acoustic effect, reaction of the surrounding tissue, presence of calcifications within the lesion, presence of vascular flow with resistance index (RI), pulsatility index (PI), and maximum velocity of the vascular flow (Vmax). Results: Among the 129 tumors included, oval shape was the most prevalent with 72 cases (56\%), 43 tumors (33\%) had an irregular shape, and only 14 (11\%) were round. As for their margin, the most prevalent was microlobulated, observed in 60 cases (46\%), followed by circumscribed (35 cases, 27\%), indistinct (19 cases, 15\%), and the less frequent margins were angled and spiculated, with $10(8 \%)$ and 5 cases (4\%), respectively. The most prevalent posterior acoustic effect was enhancement (50 cases, 39\%), followed by absence of posterior effect (38 cases, $27 \%$ ), mixed effect (23 cases, 18\%) and the least frequent was acoustic shadowing, accounting for only 18 cases (14\%). The absolute majority of the tumors, $92 \%$ lesions in total, did not present calcifications within the lesion and $89 \%$ did not have a hyperechogenic halo in the surrounding tissue, presenting with abrupt lesion limits. From all cases, $110(85 \%)$ had normal peritumoral tissue, causing no tissue thickening. In relation to the angiogenic study, most lesions had vascular flow (81 cases, 63\%). The mean PI was 1.5, RI was 0.7 and the average Vmax was $19.7 \mathrm{~cm} / \mathrm{s}$. Conclusions: From an ultrasonographic point of view, some prevalent feautres are noted, which can help in their radiogenomic determination. Oval shape, abrupt interface, microlobulated margins, posterior acoustic enhancement, absence of internal microcalcifications and hyperechogenic halo prevailed. 